\title{
EVALUATION OF LASER WELD MONITORING - A CASE STUDY
}

\author{
Paper P103
}

\author{
Ingemar Eriksson ${ }^{1}$, Alexander F. H. Kaplan ${ }^{1}$ \\ ${ }^{1}$ Luleå University of Technology, 97187 Luleå, Sweden; www.Itu.se/tfm/produktion
}

\begin{abstract}
On-line monitoring of the quality of laser welding is of interest for many industrial applications. For photodiodes the monitoring strategy usually aims at observing whether the signal exceeds a threshold. This well known technique is mainly based on empirical values and the monitoring system has to be trained for each application. For an improved understanding of the connection between the physics of the welding defect generated and the resulting monitored signal, these experiments were observed by high speed imaging, followed by evaluation and modeling. A commercial system with three detector wavelength windows was studied for nine different industrial welding applications with distinct defects. We present here selected cases for which we try to generalize the findings and to draw conclusions for the applicability of commercial monitoring systems. For example humping was clearly detected by the signal but spatter and crater formation (for overlapping $\mathrm{Zn}$-coated steel) was not. An interesting cause for welding defects is oil, grease or detergent contamination of the joint prior to welding. Monitoring defects caused in this way produced unexpected signal responses, which can be partially explained by our analysis. We summaries that an improved understanding of the signal source facilitates the reliable application of monitoring systems.
\end{abstract}

\section{Introduction}

An evaluation of a commercial online monitoring system [1] has been conducted for several different industrial cases. The monitoring is based on three photodiodes with a threshold for the signal value received from the photodiodes. Coaxial mounting of the diodes makes the mounting and alignment easy, but disturbances from the plume of ejected metal vapor from the keyhole makes monitoring less reliable. Defects are not directly correlated to the signal from emitted or reflected radiation, thus empirical rules usually need to be identified.

In front of the photodiodes there are optical band pass filters determining the observed wavelengths.
(Figure 1) The sensors are named according to their desired observations.

First, a sensor sensitive to the UV/VIS spectrum (400$600 \mathrm{~nm}$ ) is intended to observe plasma, this sensor is named the P-sensor. Next, there is a sensor with a narrow band optical filter at the Nd:YAG wavelength of $1064 \mathrm{~nm}$, intended to observe the reflected light from the welding process, in this paper named the R-sensor. Finally a sensor named T-sensor which is sensitive in the NIR region (1100-1800nm), used to observe the thermal radiation from the melt pool behind the keyhole.

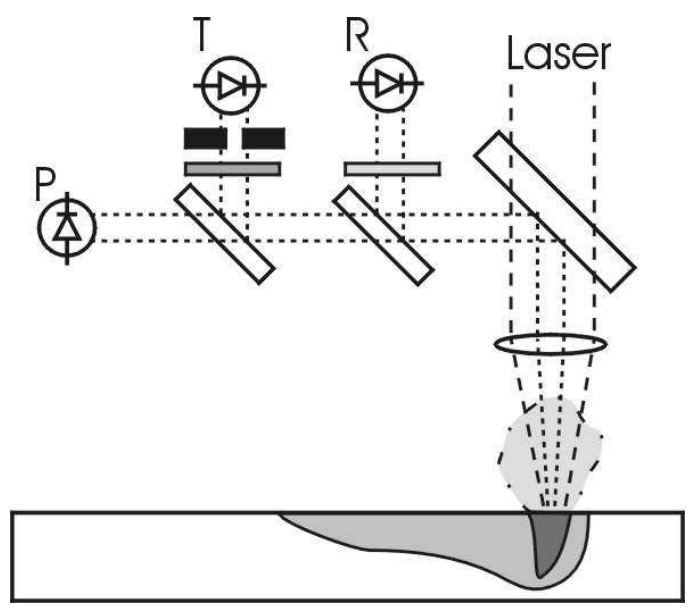

Figure 1 Photodiode monitoring setup

High temperature molten metal will radiate as described by Planck's law (1) were the radiation I is dependent on the wavelength $\lambda$ and the temperature $T$. Also the Planck constant $h$, Boltzmann constant $k$ and the speed of light $c$ is in the equation.

$\mathrm{I}(\lambda, \mathrm{T})=\left(2 h c^{2} / \lambda^{5}\right) *\left(1 /\left(\mathrm{e}^{(h c / \lambda k \mathrm{~T})}-1\right)\right.$

In front the $\mathrm{T}$-sensor an aperture can be placed to change the observed area and reduce the radiation from keyhole and plasma. The plasma created during $\mathrm{Nd}$ :YAG laser welding is less then for $\mathrm{CO}_{2}$-laser welding $\left(\mathrm{CO}_{2}\right.$ laser light is heavily absorbed by plasma). This means that most of the plume above the keyhole will be at a considerately lower temperature 
[2] than for $\mathrm{CO}_{2}$-laser welding, and thus blackbody radiation will be the dominant source of UV/VIS light. The light spectrum from a laser weld is dependant on the specific laser parameters involved and material welded, but looks similar to the schematic Figure 2 for most metals during Nd:YAG-laser welding. [3]

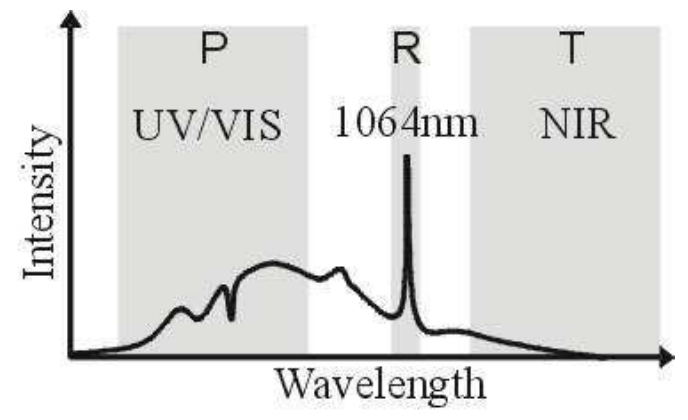

Figure 2 Radiated light from laser welding

If the emissivity of the radiating materials is assumed constant over time, it is only a scaling factor and can be ignored. The same holds for the absorption by the different optical components of the system.

The monitoring is not dependent of absolute values but on the variation of the signal and there is no need to calibrate the measurements. In the monitoring system the evaluated signal is always scaled from 0 to 10 , and there is no possibility to calibrate the measurement. This means that for threshold monitoring one or more good welds must be used as a datum for each specific welding case. This empirical method of setting the monitoring system makes the monitoring useless on short series manufacturing, and makes it hard to predict if the monitoring system will be able to detect a certain defect.

\section{The DATLAS-project}

This paper presents some of the results from the Swedish DATLAS-research project. Real industrial welding cases from eight Swedish manufactures have been investigated. Commercial online weld monitoring equipment from Precitec has been evaluated with regard to different defects. The cases have a wide spectrum of different material and different thickness, but the defects are often similar. In Table 1 some properties for the cases are presented. The type of welding is classified as $\mathrm{Nd}$ :YAG or $\mathrm{CO}_{2}$ and also whether it is keyhole $(k h)$ or conduction $(c)$ welding in continuous $(c w)$ or pulsed $(p w)$ mode. We investigated defects of the following types; Blowout $(B)$, Porosity $(P)$, Lack of fusion $(L F)$, Humping $(H)$ and Spatter $(S)$.
Table 1 Summary of different cases in DATLAS

\begin{tabular}{|c|c|c|c|}
\hline \# & Material & Type & Defect \\
\hline 1 & Titanium $1,2 \mathrm{~mm}$ & YAG,kh,cw & $\mathrm{B}, \mathrm{P}$ \\
\hline 2 & Steel $6 \mathrm{~mm}$ & Hybrid & $\mathrm{LF}$ \\
\hline 3 & Steel $12 \mathrm{~mm}$ & $\mathrm{CO}_{2}, \mathrm{kh}, \mathrm{cw}$ & $\mathrm{B}, \mathrm{LF}$ \\
\hline 4 & Stainless $2 \mathrm{~mm}$ & YAG,kh,cw & $\mathrm{LF}, \mathrm{H}$ \\
\hline 5 & Inconel $0,14 \mathrm{~mm}$ & YAG,c,pw & $\mathrm{LF}, \mathrm{S}$ \\
\hline 6 & Zn-coated $1,6 \mathrm{~mm}$ & YAG,kh,cw & $\mathrm{B}, \mathrm{P}, \mathrm{S}$ \\
\hline 7 & Steel $4 \mathrm{~mm}$ & $\mathrm{CO}_{2}, \mathrm{kh}, \mathrm{cw}$ & $\mathrm{B}, \mathrm{P}$ \\
\hline 8 & Steel 3mm & YAG,kh,pw & - \\
\hline
\end{tabular}

\section{Reflected light}

The R-sensor has a narrow $1064 \mathrm{~nm}$ band-pass optical filter mounted in front of the sensor, restricting the sensor to only Nd:YAG laser welding. Light not absorbed in the keyhole is reflected from it, but the directional complexity of this reflection is very high and almost impossible to predict. However, for a stable welding condition the amount of reflected light reaching the sensor should be fairly constant, thus if the sensor signal begins to fluctuate the welding process is changing and there is a higher probability of defects. This kind of monitoring requires a stable and well tuned welding situation, and it is difficult to determine what kind of defect has occurred. Partial or full penetration will have a dramatic effect on the reflected light, and the plume above the keyhole might also reflect some light, acting as a noise in the signal. At low power conduction mode welding, the reflected light is almost proportional to the laser power. But as soon as a keyhole is formed there is no clear correlation to physics of the welding process. In DATLAS case 5 (see Table 1), pulsed welding of 0.14 $\mathrm{mm}$ Inconel showed an increased variance of the reflected signal for bad welds.[4]. In Figure 4 the Rsignal indicates that the surface is smooth prior to the pulse, and that the surface is perpendicular to the laser beam. To achieve a sound weld there is an overlap of the pulses, thus the previous pulse produces the surface for the next pulse. In a good weld every pulse has the same behavior and the surface for each pulse is the same, Figure 3(b). This gives a small variance in the $\mathrm{R}$-sensor. If the welding becomes unstable the surface becomes rougher and the reflected light will have higher pulse to pulse variance, Figure 3(a). 
(a)

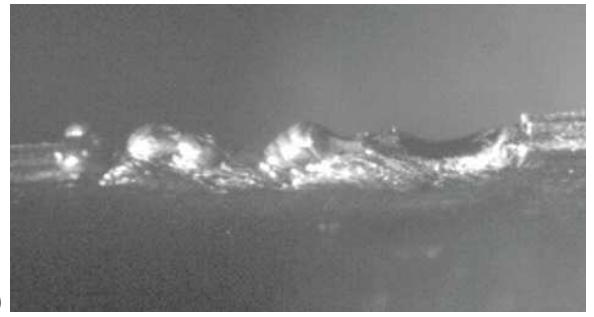

(b)

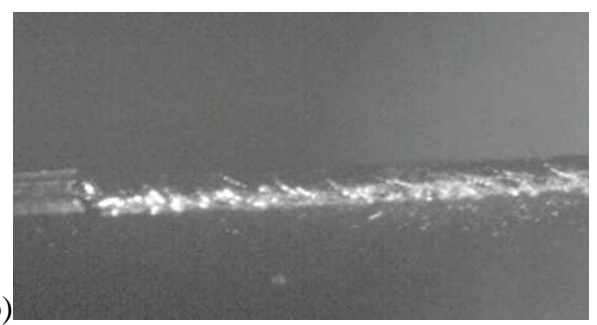

Figure 3 Pulsed edge weld of case 5

(a) Bad weld

(b) Good weld

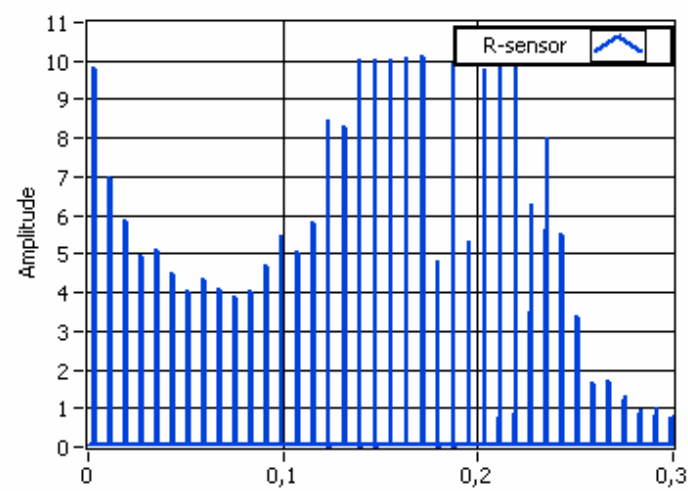

Figure 4 R-signal Amplitude vs. Time (s) from case 5: left part: stable weld, right part: disturbances

\section{T-sensor and P-sensor dynamics}

To get a deeper understanding of the origin for the Tsignal and P-signal, case 8 was created [5]. This case is long pulse welding. A $50 \%$ duty cycle and $10 \mathrm{~ms}$ pulse length creates a continuous melt pool and a keyhole is created only during the ON-period. The size of the melt pool is almost constant, as observed by the highspeed video, Figure 5. Thus the emitted light by blackbody radiation from the solid and melt pool is assumed to vary only slightly during welding. This means that the contribution from the keyhole and the plume is the difference between the signal from the $\mathrm{ON}$ and OFF periods.

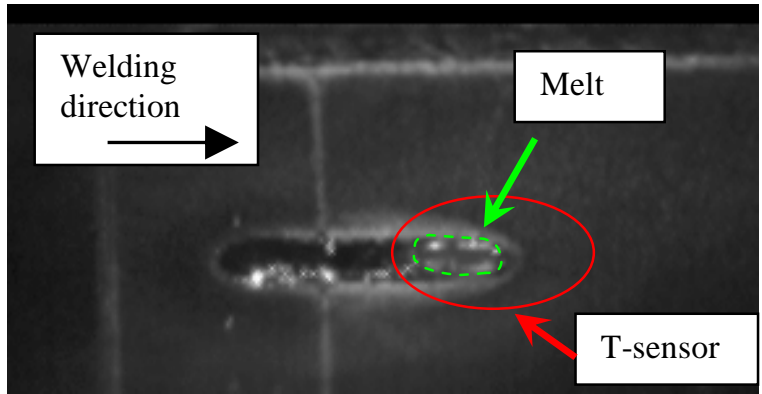

Figure 5 Melt pool size during OFF period in case 8

The signals differ considerably between the $\mathrm{ON}$ and OFF periods as can be seen in Figure 6. The OFF period has no P-signal and the T-signal is a smooth, slowly declining value. During the ON period severe fluctuations of the signals occur and the correlation of the T-signal and P-signal appears to be high.

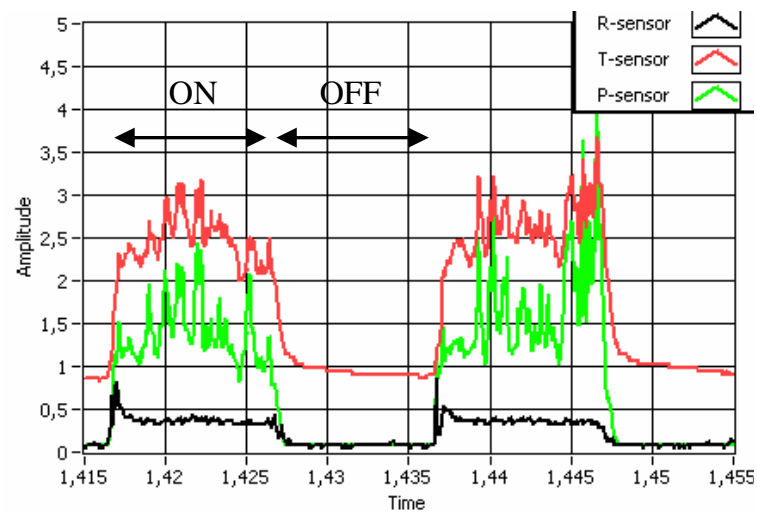

Figure 6 R-,T-,P-signals for two pulses of case 8

The difference in the $\mathrm{T}$-sensor signal between $\mathrm{ON}$ and OFF periods indicates that only $1 / 3$ of the signal comes from the melt pool and the solid surface. This is less than the previously modeled values in the DATLASproject [6]. A further investigation of the high speed video also shows that the plume is the reason for the rapid fluctuations of the signals during ON-time. The light emitted from the plume accounts for approximately $1 / 2$ of the $P$-sensor signal and $1 / 3$ of the $\mathrm{T}$-sensor signal, leaving only $1 / 3$ of the $\mathrm{T}$-sensor signal related to the keyhole itself.

\section{Surface-produced signals}

The DATLAS case 8 shows that surface emission contributes to the signal to the T-sensor. This should be useful in online monitoring, and in case 4 a clear correlation between humps in the weld and the Tsensor signal was seen. But in cases 1, 6 and 7 large blowouts cannot be detected by the T-sensor. 


\section{Humping}

In a welding case far from the normal humping regime, ( $2 \mathrm{~m} / \mathrm{min}, 3 \mathrm{~kW} \mathrm{Nd:YAG)} \mathrm{large} \mathrm{humps} \mathrm{were} \mathrm{created}$ due to improper shielding gas [6]. High speed videos show how an oxide layer starts to cover the melt pool, and the melt flow is disrupted as a large hump builds up under the oxide layer. Later on the oxide layer shrinks for a while until the next hump starts. Taking a moving average of the T-sensor signal shows the correlation between humps and signal. The humping phenomenon has a frequency of less than $10 \mathrm{~Hz}$ in this case.

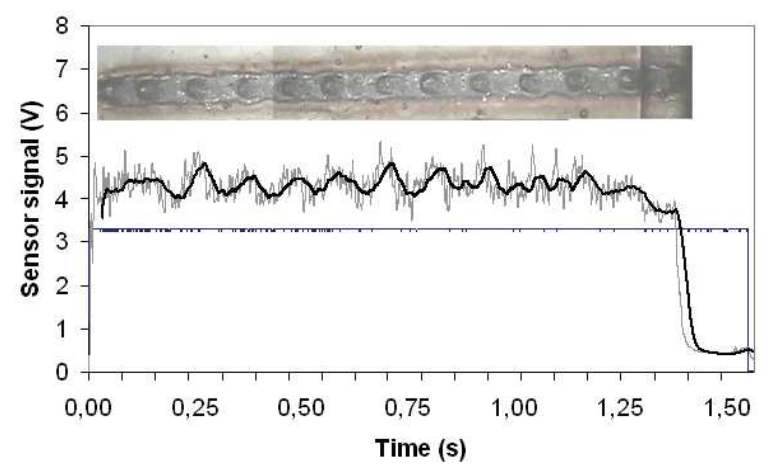

Figure 7 Signal and image from humping in case 4

Even though the images of the welding do not indicate it, we can see additional peaks at around 0.8-1.2 $\mathrm{s}$ in Figure 7. This could be due to the process or by aliasing in the $\mathrm{A} / \mathrm{D}$-converter as there is no antialiasing filter in the hardware.

\section{Blowouts and pores}

Several cases in the DATLAS-project had problems with pores and blowouts, cases 1, 6 and 7 revealed that the source of the problem was contamination leading to gas in the melt pool. The gas is created by vaporization of zinc in case 6 , grease and oil in case 7 , and in case 1 the problem was residue of detergents after cleaning the titanium. Pores are formed by small gas bubbles, but if a large gas bubble reaches the surface a blowout will eject molten metal from the weld.

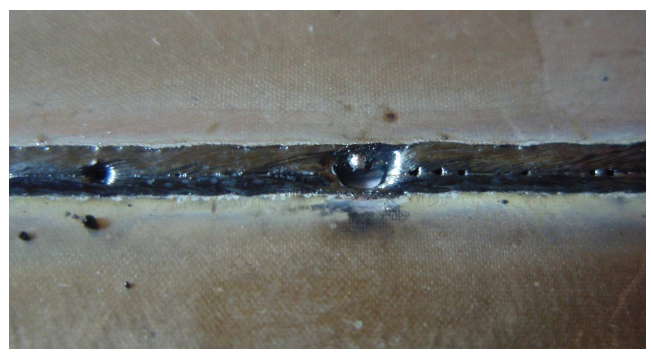

Figure 8 Top-view of case 6, Zn-coated with holes
Figure 8 shows the effect of holes of different size when they reach the surface, this has been further categorized in [7]. The time for a gas bubble to explode is relatively short, Large ones throwing out metal may have a duration of a few $\mathrm{ms}$, small ones which only produce surface pores are of sub-ms duration.

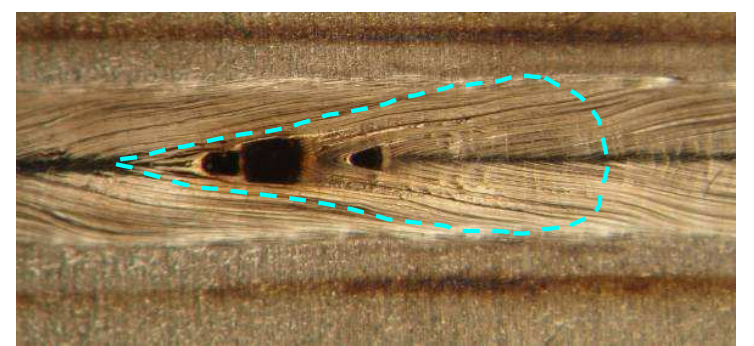

Figure 9 Top-view of case 1 with holes

Small gas bubbles often reach the surface at the end of the melt pool. This is easy to see in the high speed video, and is explained by the fact that the melt pool solidifies towards the center of the weld. In Figure 9 the ripples in the weld indicate the shape of the melt pool when a pore was formed. This shows that pores and blowouts often are created outside the keyhole area. Unless the creation of the gas affects the keyhole, it is impossible to detect blowouts with the R-sensor. In a large melt pool pores created at the end of the pool are often outside the monitored area and impossible to detect by $\mathrm{T}$-sensor.

Sub-surface pores created by gas bubbles trapped inside the melt pool can be hard to detect (but not impossible, e.g. if the melt surface is lifted up). In Figure 10 a cut along the welding direction shows the amount of porosity possible in a weld without any surface pores. The only indication of pores was the enlarged weld crown. No change of the sensor signals compared to a weld without pores was observed, thus online monitoring could not detect the pores.

Contamination of oil and an unfavorable joint geometry created the large amount of pores in this weld from DATLAS case 7.

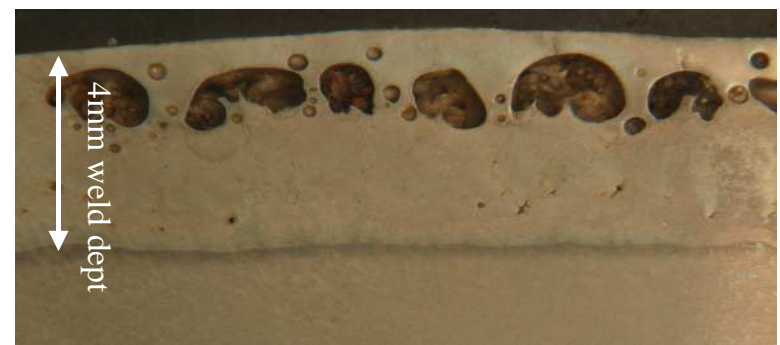

Figure $10 \mathrm{Cut}$ along the welding direction with pores, case 7 
All three cases with pore and blowout defects have a joint geometry that captures the gas from material with a low evaporation temperature.

A closer investigation of a single blowout from case 6 will now be presented. The weld is a standard automotive zinc-coated lap joint. Blowouts are created due to zero gap and deliberate non optimal welding parameters, Figure 11. Two high-speed cameras were used, one at 45 degrees inclination with an illumination laser to film the melt pool at $2500 \mathrm{fps}$. The illumination cancels out the emissions from the plume and melt pool, giving clear photos. Another camera at 0 degrees inclination filming the plume without illumination is running at $40000 \mathrm{fps}$ giving a good view of the plume dynamics.

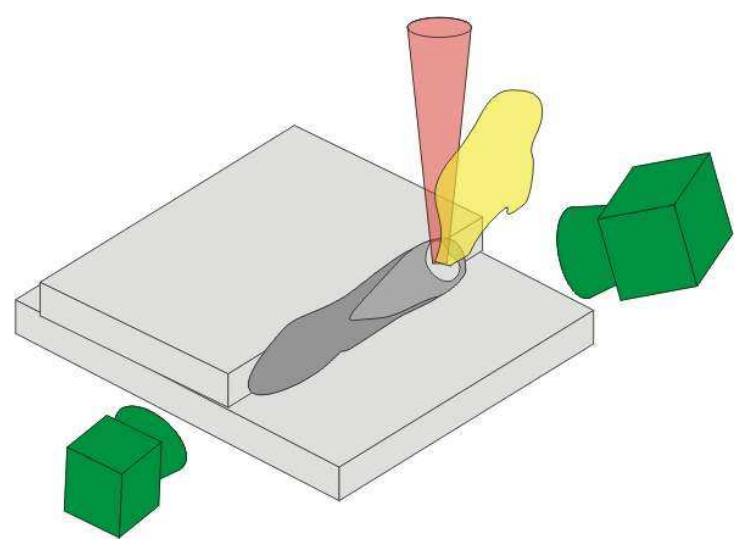

Figure 11. Experimental setup case 6

Figure 12 shows one frame from the plume monitoring camera. The light emitting plume is fast moving and has stochastic behavior. The motions of the plume can be correlated to the signal amplitude of the T-sensor and $\mathrm{P}$-sensor, explaining most of the variations in these signals. More on this subject will be published soon.

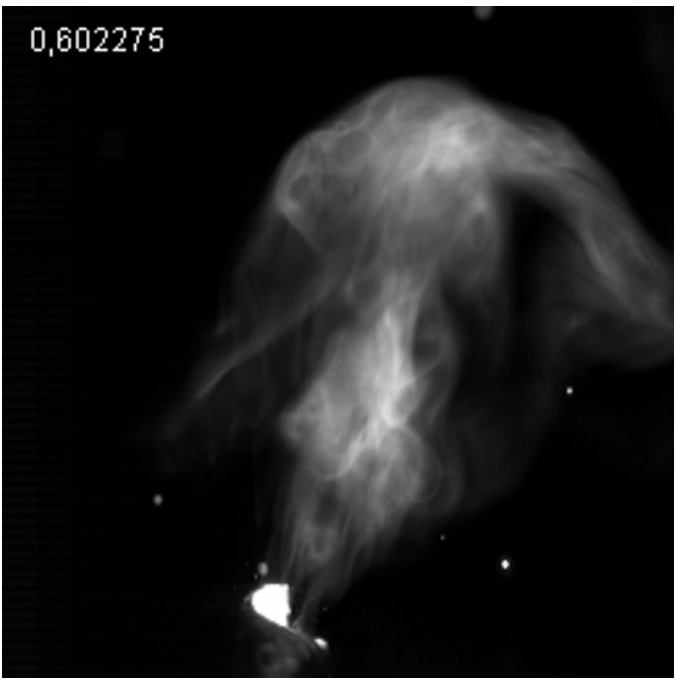

Figure 12 Single frame of the plume, case 6

In Figure 14 successive frames from the tilted camera are shown. The frame rate of $2500 \mathrm{fps}$ gives a duration of $2 \mathrm{~ms}$ for the five frames. A blowout starts far behind the keyhole visible to the right in the images and ejects molten metal from the weld, leaving a big hole behind. Figure 13 shows the signals during this event with the blowout occurring at $0,618-0,620$.

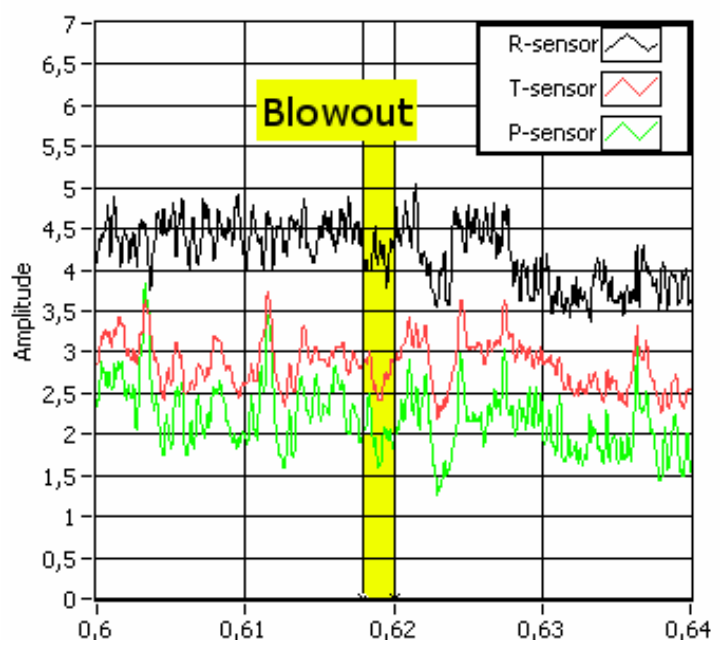

Figure 13 Signal during a blowout, case 6

There is no distinct change of signal amplitude during the blowout. The dip in the signals amplitude at 0.623 is due to a large droplet from the blowout passing close above the keyhole, a unique event of this particular blowout. The very noisy signal created by the plume is a main contribution to the $\mathrm{P}$ - and $\mathrm{T}$-sensor signal and makes it impossible for the online monitoring system to detect blowouts. 

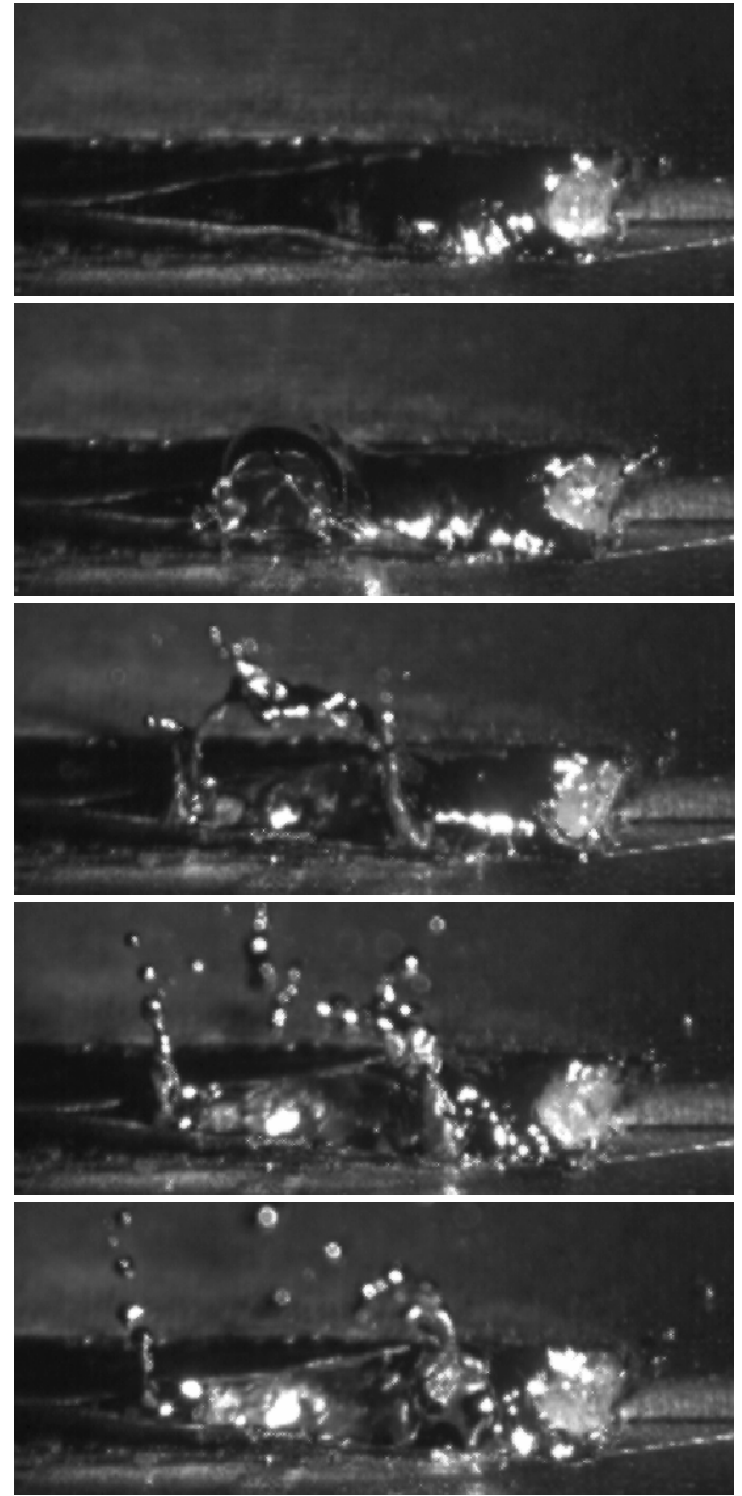

Figure 14 A high speed image sequence (2500 fps) of a blowout event with the melt pool and keyhole (right), case 6 .

\section{Conclusions}

On-line monitoring of welding defects by photodiodes requires empirical correlations or rules. High speed imaging is a powerful tool in evaluating online laser weld monitoring. The possibility to see the motion of the weld is helpful in judging the possibility to detect (and even to explain) a particular defect. The high frequency behavior of the plume effectively masks other fast events and reduces the effectiveness of monitoring. This effect has been found to a varying extent in all the DATLAS cases.
From the different cases studied a broad knowledge of the behavior of the sensor signals was obtained, which has, or will be, documented in a series of publications [1],[4]-[8].

The conclusions of the evaluation of the different cases in the DATLAS-project can be summarized as:

- The reflected (R-sensor) light from a keyhole is hard to predict, but during pulsed welding it can often be useful to judge the quality of the weld in a different way than the $\mathrm{T}$ - and $\mathrm{P}$-sensors.

- The T-sensor and the P-sensor have a high correlation to the fluctuation of the observed plume above the keyhole, and thus often deliver a similar signal.

- Pores can be formed due to captured gas from boiling detergent, grease or zinc. If the enclosed gas bubble reaches the surface there is a blow-out. However, the blowout was often not detectable, as explained above.

- Slow melt surface activity (e.g. humping) can be observed by the T-sensor while blowouts are rapid events concealed by the fluctuations of the plume.

\section{Acknowledgements}

This work was funded by VINNOVA, The Swedish Innovation Agency, projects no. 2006-00668, 200502895, 2006-00563, and by the Kempe foundation. The authors are also grateful to Precitec $\mathrm{GmbH}$ for their support.

\section{References}

[1] Norman, P., Engström, H. \& Kaplan, A. (2007) State-of-the-art of monitoring and imaging of laser welding defects, in Proceedings of the NOLAMPConference, Lappeenranta, Finland

[2] Kawahito,Y., Kinoshita,M., Matsumoto,N. \& Katayama,S. (2009) Visualization of refraction and attenuation of near-infrared laser beam due to laserinduced plume, J. Laser Appl. Vol.21, No.2, pp.96-101

[3] Aalderink, B.J., Aarts, R.G.K.M., Jonker, J.B, \& Meijer, J, (2005) Weld Plume Emissions During Nd:YAG Laser Welding, in Proceedings of the WLTconference on Lasers in Manufacturing, Munich, Germany, p.413-417

[4] Olsson, R., Eriksson, I., Powell, J. \& Kaplan, A.F.H. (2009) Pulsed laser weld quality monitoring by the statistical analysis of reflected light., in 
Proceedings of the WLT-conference on Lasers in Manufacturing, Munich, Germany, (in press)

[5] Eriksson, E. A. I., Norman, P.,\& Kaplan, A. F. H. (2009) Basic study of photodiode signals from laser welding emissions, in Proceedings of the NOLAMPconference, Copenhagen, Denmark, (in press)

[6] Norman, P., Engström, H. \& Kaplan, A. F. H. (2008) Theoretical analysis of photodiode monitoring of laser welding defects by imaging combined with modelling, J. Phys. D: Appl. Phys. 41

[7] Norman, P., Eriksson, I. \& Kaplan, A. F. H. (2009) Monitoring laser beam welding of zinc coated sheet metal to analyse the defects occurring, in Proceedings of the NOLAMP-conference, Copenhagen, Denmark, (in press)

[8] Norman, P., Karlsson, J. \& Kaplan, A. F.H. (2009) Monitoring undercut, blowouts and root sagging during laser beam welding, in Proceedings of the WLT-conference on Lasers in Manufacturing, Munich, Germany (in press)

\section{Meet the Author}

Ingemar Eriksson was born in Östersund, Sweden in 1980. He studied Electrical Engineering at the Mid Sweden University from 2001 to 2005. After three years in industry he resumed the academics and started on his PhD-studies in laser welding at Luleå University of Technology in 2008. He is currently working with online process monitoring of laser welding.

Alexander Kaplan was born in Vienna, Austria in 1967, employed as researcher at Vienna TU from 1989 until 2000, receiving a $\mathrm{PhD}$-degree in 1994. After a post-doc year at Osaka University, Japan, from 2002 on he has been appointed as professor and head of division on manufacturing at Luleå University of Technology, Sweden. His research focus comprises laser materials processing, in particular process modelling, laser welding and hybrid welding. 